JOURNAL OF

FUNCTION SPACES AND APPLICATIONS

Volume 5, Number 2 (2007), 123-132
(C) 2007, Scientific Horizon http://www.jfsa.net

\title{
A homogeneity property for Besov spaces
}

\section{António M. Caetano, Sofia Lopes and Hans Triebel}

(Communicated by Victor Burenkov)

2000 Mathematics Subject Classification. 46E35.

Keywords and phrases. Homogeneity, Besov spaces, differences.

Abstract. A homogeneity property for some Besov spaces $B_{p, q}^{s}$ is proved. An analogous property for some $F_{p, q}^{s}$ spaces is already known.

\section{Introduction}

In the present note we prove the so-called homogeneity property for some Besov spaces on $\mathbb{R}^{n}$. We show that for $0<p, q \leq \infty$ and $s>\sigma_{p}$,

$$
\left\|f(\lambda \cdot)\left|B_{p, q}^{s}\left(\mathbb{R}^{n}\right)\left\|\sim \lambda^{s-\frac{n}{p}}\right\| f\right| B_{p, q}^{s}\left(\mathbb{R}^{n}\right)\right\|,
$$

for all $0<\lambda \leq 1$ and all

$$
f \in B_{p, q}^{s}\left(\mathbb{R}^{n}\right) \quad \text { with } \quad \operatorname{supp} f \subset\left\{x \in \mathbb{R}^{n}:|x| \leq \lambda\right\} .
$$

A corresponding property for the spaces $F_{p, q}^{s}\left(\mathbb{R}^{n}\right)$ may be found in $[9$, Corollary 5.16, p. 66]. It is of some use in connection with refined localization, non-smooth atoms, scaling properties and pointwise multipliers. We refer to [10, Section 4.2.2]. Here we prove this property for the spaces $B_{p, q}^{s}\left(\mathbb{R}^{n}\right)$, with $s>\sigma_{p}$. For the particular case where $\sigma_{p}<s<n / p$ this property can be obtained via atomic decompositions. This was the starting point for the main result presented in this note. 
This note is organised as follows: firstly we give the necessary definitions; secondly we deal with equivalent quasi-norms for the elements of certain subspaces of $B_{p, q}^{s}\left(\mathbb{R}^{n}\right)$; finally we present the main result referred above.

\section{Preliminaries}

First we introduce some standard notation and useful definitions. As usual, $\mathbb{N}$ denotes the set of all natural numbers, $\mathbb{R}^{n}, n \in \mathbb{N}$, stands for the $n$-dimensional real Euclidean space and $\mathbb{R}=\mathbb{R}^{1}$. We use the symbol "§" in

$$
a_{k} \lesssim b_{k} \quad \text { or } \quad \phi(r) \lesssim \psi(r)
$$

always to mean that there is a positive number $c_{1}$ such that

$$
a_{k} \leq c_{1} b_{k} \quad \text { or } \quad \phi(r) \leq c_{1} \psi(r)
$$

for all admitted values of the discrete variable $k$ or the continuous variable $x$, where $\left(a_{k}\right)_{k},\left(b_{k}\right)_{k}$ are non-negative sequences and $\phi, \psi$ are nonnegative functions. We use the equivalence " $\sim$ " in

$$
a_{k} \sim b_{k} \quad \text { or } \quad \phi(r) \sim \psi(r)
$$

for

$$
a_{k} \lesssim b_{k} \quad \text { and } \quad b_{k} \lesssim a_{k} \quad \text { or } \quad \phi(r) \lesssim \psi(r) \quad \text { and } \quad \psi(r) \lesssim \phi(r)
$$

As usual $\mathcal{S}\left(\mathbb{R}^{n}\right)$ denotes the Schwartz space of all complex-valued rapidly decreasing infinitely differentiable functions on $\mathbb{R}^{n}$ equipped with the usual topology, and $\mathcal{S}^{\prime}\left(\mathbb{R}^{n}\right)$ denotes its topological dual, the space of all tempered distributions on $\mathbb{R}^{n}$. Furthermore, $L_{p}\left(\mathbb{R}^{n}\right)$, with $0<p \leq \infty$, stands for the usual quasi-Banach space with respect to the Lebesgue measure, quasinormed by

$$
\left\|f \mid L_{p}\left(\mathbb{R}^{n}\right)\right\|:=\left(\int_{\mathbb{R}^{n}}|f(x)|^{p} d x\right)^{\frac{1}{p}}
$$

with the appropriate modification if $p=\infty$.

We use the standard abbreviations

$$
\bar{p}:=\max (1, p) \quad \text { and } \quad \sigma_{p}:=n\left(\frac{1}{p}-1\right)_{+}=n\left(\frac{1}{\min \{1, p\}}-1\right) .
$$

As usually, "domain" stands for "open set".

Next, we recall the definition of differences of functions. If $f$ is an arbitrary 
complex-valued function on $\mathbb{R}^{n}, h \in \mathbb{R}^{n}$ and $M \in \mathbb{N}$, then

$$
\left(\Delta_{h}^{M} f\right)(x):=\sum_{j=0}^{M}\left(\begin{array}{c}
M \\
j
\end{array}\right)(-1)^{M-j} f(x+j h), \quad x \in \mathbb{R}^{n}
$$

where $\left(\begin{array}{c}M \\ j\end{array}\right)$ are the binomial coefficients.

Definition 1. Let

$$
0<p, q \leq \infty \quad \text { and } \quad \sigma_{p}<s<M \in \mathbb{N} .
$$

Then $B_{p, q}^{s}\left(\mathbb{R}^{n}\right)$ is the collection of all $f \in L_{p}\left(\mathbb{R}^{n}\right)$ such that

(6)

$$
\left\|f\left|B_{p, q}^{s}\left(\mathbb{R}^{n}\right)\left\|_{M}:=\right\| f\right| L_{p}\left(\mathbb{R}^{n}\right)\right\|+\left(\int_{\mathbb{R}^{n}}|h|^{-s q}\left\|\Delta_{h}^{M} f \mid L_{p}\left(\mathbb{R}^{n}\right)\right\|^{q} \frac{d h}{|h|^{n}}\right)^{1 / q}
$$

(with the usual modification if $q=\infty$ ) is finite.

Remark 2. The above spaces are quasi-Banach spaces. They have a substantial history. The classical spaces, which means $1 \leq p, q \leq \infty$, $s>0$, go back to S.M. Nikol'skij in the 1950s $(q=\infty)$ and O.V. Besov around 1960. These spaces have been considered afterwards in great detail especially by the Russian school. This may be found in [5] and [1]. At least in this specification they are also denoted as Nikol'skij-Besov spaces. The extension to $s<0$ (what is not of interest for our purpose) and to $p<1$ (which is subject of the later considerations) was first done by J. Peetre at the end of the 1960s and the early 1970s in terms of Fourier-analytical definitions. We refer to [6]. Systematic studies of these spaces may be found in $[7,8]$. The first chapters both of [8] and [10] are surveys (entitled How to measure smoothness) where one finds the history of these spaces. The spaces $B_{p, q}^{s}\left(\mathbb{R}^{n}\right)$ are nowadays often introduced in terms of Fourieranalytical decompositions. If $p, q, s$ are restricted by (5) then these spaces coincide with the spaces as introduced in Definition 1. A proof may be found in [7, Section 2.5.12, Theorem, Remark 3, Corollary, pp. 110-114] covering also that

$$
B_{\infty, \infty}^{s}\left(\mathbb{R}^{n}\right)=\mathcal{C}^{s}\left(\mathbb{R}^{n}\right), \quad s>0,
$$

are the usual Hölder-Zygmund spaces, characterized by

$$
\sup _{x \in \mathbb{R}^{n}}|f(x)|+\sup _{x \in \mathbb{R}^{n}, 0 \neq h \in \mathbb{R}^{n}}|h|^{-s}\left|\Delta_{h}^{M} f(x)\right|<\infty
$$

where again $s<M \in \mathbb{N}$. The quasi-norms in (6) are equivalent to each other for different values of $M$ with $0<s<M \in \mathbb{N}$. This justifies our omission of the subscript $M$ in the sequel. 


\section{Equivalent quasi-metrics}

We collect further notation.

Definition 3. Let $\Omega$ be a domain in $\mathbb{R}^{n}$. Let $s \in \mathbb{R}, 0<p \leq \infty$, $0<q \leq \infty$.

(i) Then $L_{p}(\Omega)$ is the collection of all complex-valued Lebesgue measurable functions in $\Omega$ such that

$$
\left\|f \mid L_{p}(\Omega)\right\|:=\left(\int_{\Omega}|f(x)|^{p} d x\right)^{\frac{1}{p}}
$$

(with the usual modification if $p=\infty$ ) is finite.

(ii) Then $B_{p, q}^{s}(\Omega)$ is the collection of all $f \in D^{\prime}(\Omega)$ such that there is a $g \in B_{p, q}^{s}\left(\mathbb{R}^{n}\right)$ with $\left.g\right|_{\Omega}=f$. Furthermore,

$$
\left\|f\left|B_{p, q}^{s}(\Omega)\|:=\inf \| g\right| B_{p, q}^{s}\left(\mathbb{R}^{n}\right)\right\|,
$$

where the infimum is taken over all $g \in B_{p, q}^{s}\left(\mathbb{R}^{n}\right)$ such that its restriction $\left.g\right|_{\Omega}$ to $\Omega$ coincides in $D^{\prime}(\Omega)$ with $f$.

(iii) Then $\widetilde{B}_{p, q}^{s}(\bar{\Omega})$ is the closed subspace of $B_{p, q}^{s}\left(\mathbb{R}^{n}\right)$ given by

$$
\widetilde{B}_{p, q}^{s}(\bar{\Omega}):=\left\{f \in B_{p, q}^{s}\left(\mathbb{R}^{n}\right): \operatorname{supp} f \subset \bar{\Omega}\right\} .
$$

Remark 4. These spaces are quasi-Banach spaces. Function spaces on domains have also been studied in detail from the very beginning of the theory of function spaces in 1950s and 1960s. We refer to the books in Remark 2 and to [7, Chapter 3],[8, Chapter 5] and [9, Section 5].

Proposition 5. Let $\Omega$ be a bounded domain in $\mathbb{R}^{n}$ and

$$
0<p \leq \infty, \quad 0<q \leq \infty, \quad \sigma_{p}<s<M \in \mathbb{N}
$$

Then

$$
\left\|f \mid B_{p, q}^{s}\left(\mathbb{R}^{n}\right)\right\| \sim\left(\int_{\mathbb{R}^{n}}|h|^{-s q}\left\|\Delta_{h}^{M} f \mid L_{p}\left(\mathbb{R}^{n}\right)\right\|^{q} \frac{d h}{|h|^{n}}\right)^{1 / q}
$$

for all $f \in \widetilde{B}_{p, q}^{s}(\bar{\Omega})$ (equivalent quasi-norms), with the usual modification if $q=\infty$.

Proof. We consider

$$
B_{R}=\left\{x \in \mathbb{R}^{n}:|x|<R\right\}
$$


with $\bar{\Omega} \subset B_{R}$. Let $0<q<\infty$. We recall that by Definition 1 we have (6) for the full space $B_{p, q}^{s}\left(\mathbb{R}^{n}\right)$. If $p<1$, as $s>\sigma_{p}$, we get by well-known embedding theorems that $B_{p, q}^{s}\left(\mathbb{R}^{n}\right) \subset L_{1}\left(\mathbb{R}^{n}\right)$. Consequently, by (6), we obtain

(12) $\left\|f\left|L_{1}\left(\mathbb{R}^{n}\right)\left\|+\left(\int_{\mathbb{R}^{n}}|h|^{-s q}\left\|\Delta_{h}^{M} f \mid L_{p}\left(\mathbb{R}^{n}\right)\right\|^{q} \frac{d h}{|h|^{n}}\right)^{1 / q} \lesssim\right\| f\right| B_{p, q}^{s}\left(\mathbb{R}^{n}\right)\right\|$.

If $f \in \widetilde{B}_{p, q}^{s}(\bar{\Omega})$, then

$$
\left\|f\left|L_{p}\left(\mathbb{R}^{n}\right)\|=\| f\right| L_{p}\left(B_{R}\right)\right\| \lesssim\left\|f\left|L_{1}\left(B_{R}\right)\|=\| f\right| L_{1}\left(\mathbb{R}^{n}\right)\right\| .
$$

Thus, using (6), we obtain, for $p<1$,

(14) $\left\|f\left|B_{p, q}^{s}\left(\mathbb{R}^{n}\right)\|\lesssim\| f\right| L_{1}\left(\mathbb{R}^{n}\right)\right\|+\left(\int_{\mathbb{R}^{n}}|h|^{-s q}\left\|\Delta_{h}^{M} f \mid L_{p}\left(\mathbb{R}^{n}\right)\right\|^{q} \frac{d h}{|h|^{n}}\right)^{1 / q}$.

Hence, for all $f \in \widetilde{B}_{p, q}^{s}(\bar{\Omega})$ and $0<p \leq \infty$,

(15) $\left\|f\left|B_{p, q}^{s}\left(\mathbb{R}^{n}\right)\|\sim\| f\right| L_{\bar{p}}\left(\mathbb{R}^{n}\right)\right\|+\left(\int_{\mathbb{R}^{n}}|h|^{-s q}\left\|\Delta_{h}^{M} f \mid L_{p}\left(\mathbb{R}^{n}\right)\right\|^{q} \frac{d h}{|h|^{n}}\right)^{1 / q}$.

It remains to prove that there is a positive number $c$ such that

$$
\left\|f \mid L_{\bar{p}}\left(\mathbb{R}^{n}\right)\right\| \leq c\left(\int_{\mathbb{R}^{n}}|h|^{-s q}\left\|\Delta_{h}^{M} f \mid L_{p}\left(\mathbb{R}^{n}\right)\right\|^{q} \frac{d h}{|h|^{n}}\right)^{1 / q},
$$

for all $f \in \widetilde{B}_{p, q}^{s}(\bar{\Omega})$. Assuming that there is no such $c$, then for every $j \in \mathbb{N}$ one finds a function $f_{j} \in \widetilde{B}_{p, q}^{s}(\bar{\Omega})$ which can be normalised such that

$$
1=\left\|f_{j} \mid L_{\bar{p}}\left(\mathbb{R}^{n}\right)\right\|>j\left(\int_{\mathbb{R}^{n}}|h|^{-s q}\left\|\Delta_{h}^{M} f_{j} \mid L_{p}\left(\mathbb{R}^{n}\right)\right\|^{q} \frac{d h}{|h|^{n}}\right)^{1 / q} .
$$

Hence

$$
\begin{aligned}
\left\|\left.f_{j}\right|_{B_{R}} \mid B_{p, q}^{s}\left(B_{R}\right)\right\| \leq & \left\|f_{j} \mid B_{p, q}^{s}\left(\mathbb{R}^{n}\right)\right\| \\
& \\
& \quad\left\|f_{j} \mid L_{\bar{p}}\left(\mathbb{R}^{n}\right)\right\| \\
& +\left(\int_{\mathbb{R}^{n}}|h|^{-s q}\left\|\Delta_{h}^{M} f_{j} \mid L_{p}\left(\mathbb{R}^{n}\right)\right\|^{q} \frac{d h}{|h|^{n}}\right)^{1 / q} \\
& <1+\frac{1}{j} \leq 2 .
\end{aligned}
$$


As $\left\{\left.f_{j}\right|_{B_{R}}\right\}_{j \in \mathbb{N}}$ is bounded in $B_{p, q}^{s}\left(B_{R}\right)$ and the embedding of $B_{p, q}^{s}\left(B_{R}\right)$ into $L_{\bar{p}}\left(B_{R}\right)$ is compact (cf. e.g. [10, Theorem 1.97, Proposition 4.6] and the references given there), $\left\{\left.f_{j}\right|_{B_{R}}\right\}_{j \in \mathbb{N}}$ is precompact in $L_{\bar{p}}\left(B_{R}\right)$. We may assume that, for some $f \in L_{\bar{p}}\left(B_{R}\right)$,

$$
\left.f_{j}\right|_{B_{R}} \rightarrow f \quad \text { in } \quad L_{\bar{p}}\left(B_{R}\right)
$$

and, consequently,

$$
\left\|f \mid L_{\bar{p}}\left(B_{R}\right)\right\|=1
$$

For $j, j^{\prime} \in \mathbb{N}$, using (6), we get

$$
\begin{aligned}
& \left\|f_{j}-f_{j^{\prime}} \mid B_{p, q}^{s}\left(\mathbb{R}^{n}\right)\right\| \\
& \sim\left\|f_{j}-f_{j^{\prime}} \mid L_{\bar{p}}\left(\mathbb{R}^{n}\right)\right\|+\left(\int_{\mathbb{R}^{n}}|h|^{-s q}\left\|\Delta_{h}^{M}\left(f_{j}-f_{j^{\prime}}\right) \mid L_{p}\left(\mathbb{R}^{n}\right)\right\|^{q} \frac{d h}{|h|^{n}}\right)^{1 / q} .
\end{aligned}
$$

Using (19) and the fact that $f_{j} \in \widetilde{B}_{p, q}^{s}(\bar{\Omega})$, we obtain

(21) $\left\|f_{j}-f_{j^{\prime}}\left|L_{\bar{p}}\left(\mathbb{R}^{n}\right)\|=\| f_{j}\right|_{B_{R}}-\left.f_{j^{\prime}}\right|_{B_{R}} \mid L_{\bar{p}}\left(B_{R}\right)\right\| \rightarrow 0 \quad$ as $\quad j, j^{\prime} \rightarrow \infty$.

By (17) we get

$$
\begin{aligned}
& \left(\int_{\mathbb{R}^{n}}|h|^{-s q}\left\|\Delta_{h}^{M}\left(f_{j}-f_{j^{\prime}}\right) \mid L_{p}\left(\mathbb{R}^{n}\right)\right\|^{q} \frac{d h}{|h|^{n}}\right)^{1 / q} \\
& \lesssim\left(\int_{\mathbb{R}^{n}}|h|^{-s q}\left\|\Delta_{h}^{M} f_{j} \mid L_{p}\left(\mathbb{R}^{n}\right)\right\|^{q} \frac{d h}{|h|^{n}}\right)^{1 / q} \\
& \quad+\left(\int_{\mathbb{R}^{n}}|h|^{-s q}\left\|\Delta_{h}^{M} f_{j^{\prime}} \mid L_{p}\left(\mathbb{R}^{n}\right)\right\|^{q} \frac{d h}{|h|^{n}}\right)^{1 / q} \\
& <\frac{1}{j}\left\|f_{j}\left|L_{\bar{p}}\left(\mathbb{R}^{n}\right)\left\|+\frac{1}{j^{\prime}}\right\| f_{j^{\prime}}\right| L_{\bar{p}}\left(\mathbb{R}^{n}\right)\right\| \\
& =\frac{1}{j}+\frac{1}{j^{\prime}} \rightarrow 0 \quad \text { as } \quad j, j^{\prime} \rightarrow \infty .
\end{aligned}
$$

Hence $\left\{f_{j}\right\}_{j \in \mathbb{N}}$ converges in $B_{p, q}^{s}\left(\mathbb{R}^{n}\right)$ to, say, g. As all $f_{j}$ are elements of $\widetilde{B}_{p, q}^{s}(\bar{\Omega})$, which is a closed subspace of $B_{p, q}^{s}\left(\mathbb{R}^{n}\right)$, we get $g \in \widetilde{B}_{p, q}^{s}(\bar{\Omega})$. As

$$
\left\|f_{j}-g \mid B_{p, q}^{s}\left(\mathbb{R}^{n}\right)\right\| \rightarrow 0 \quad \text { as } \quad j \rightarrow \infty
$$


we get

$$
\left\|\left.f_{j}\right|_{B_{R}}-\left.g\right|_{B_{R}}\left|L_{\bar{p}}\left(B_{R}\right)\|=\| f_{j}-g\right| L_{\bar{p}}\left(\mathbb{R}^{n}\right)\right\| \rightarrow 0 \quad \text { as } \quad j \rightarrow \infty
$$

and $g=f$ according to (19). Thus,

$$
\left\|\left.g\right|_{B_{R}}\left|L_{\bar{p}}\left(B_{R}\right)\|=\| f_{j}\right|_{B_{R}} \mid L_{\bar{p}}\left(B_{R}\right)\right\|=1 .
$$

Now (assuming $q<\infty$ )

$$
\begin{aligned}
& \int_{\mathbb{R}^{n}}|h|^{-s q}\left\|\Delta_{h}^{M} g \mid L_{p}\left(\mathbb{R}^{n}\right)\right\|^{q} \frac{d h}{|h|^{n}} \\
& \quad \lesssim \int_{\mathbb{R}^{n}}|h|^{-s q}\left\|\Delta_{h}^{M}\left(g-f_{j}\right)\left|L_{p}\left(\mathbb{R}^{n}\right)\left\|^{q} \frac{d h}{|h|^{n}}+\int_{\mathbb{R}^{n}}|h|^{-s q}\right\| \Delta_{h}^{M} f_{j}\right| L_{p}\left(\mathbb{R}^{n}\right)\right\|^{q} \frac{d h}{|h|^{n}} \\
& \quad \lesssim\left\|g-f_{j} \mid B_{p, q}^{s}\left(\mathbb{R}^{n}\right)\right\|^{q}+\frac{1}{j^{q}} \rightarrow 0 \quad \text { as } \quad j \rightarrow \infty .
\end{aligned}
$$

Therefore

$$
\int_{\mathbb{R}^{n}}|h|^{-s q}\left\|\Delta_{h}^{M} g \mid L_{p}\left(\mathbb{R}^{n}\right)\right\|^{q} \frac{d h}{|h|^{n}}=0 .
$$

If $q=\infty$ one has to modify the argument in the usual way. Let

$$
C=\left\{y \in \mathbb{R}^{n}: g(y) \neq 0\right\} \cap B_{R} .
$$

We wish to show that $|C|=0$. We prove it by contradiction. We suppose that $|C|>0$. By $(25),\left\|\Delta_{h}^{M} g \mid L_{p}\left(\mathbb{R}^{n}\right)\right\|=0$ for almost every $h \in \mathbb{R}^{n}$. Let $A$ denote a subset of $\mathbb{R}^{n}$ with $|A|=0$ such that $\left\|\Delta_{h}^{M} g \mid L_{p}\left(\mathbb{R}^{n}\right)\right\|=0$, for all $h \in A^{c}$. We fix $h \in A^{c}$ such that $|h|>2 R$. As $\left\|\Delta_{h}^{M} g \mid L_{p}\left(\mathbb{R}^{n}\right)\right\|=0$, there is $D_{h} \subset \mathbb{R}^{n}$ with $\left|D_{h}\right|=0$ such that $\left(\Delta_{h}^{M} g\right)(x)=0$, for all $x \in D_{h}^{c}$. As $|C|>0$ and $\left|D_{h}\right|=0$, there are points $x \in D_{h}^{c} \cap C$. Considering such an $x$, we get on the one hand that

$$
\left(\Delta_{h}^{M} g\right)(x)=0
$$

because $h \in A^{c}$ and $x \in D_{h}^{c}$. On the other hand, by (26) and using the hypothesis that $x \in C \subset B_{R}$ and $|h|>2 R$, we obtain

$$
\left(\Delta_{h}^{M} g\right)(x)=\sum_{j=0}^{M}\left(\begin{array}{c}
M \\
j
\end{array}\right)(-1)^{M-j} g(x+j h)=(-1)^{M} g(x) \neq 0
$$


which contradicts (27). Hence $|C|=0$, i.e., $\left.g\right|_{B_{R}}=0$ almost everywhere. This contradicts $(24)$.

\section{Homogeneity}

Finally we present and prove the main result. Recall that $B_{\lambda}=\{x \in$ $\left.\mathbb{R}^{n}:|x|<\lambda\right\}$.

Theorem 6. Let

$$
0<p \leq \infty, \quad 0<q \leq \infty, \quad s>\sigma_{p} .
$$

Then, for all $0<\lambda \leq 1$ and $f \in \widetilde{B}_{p, q}^{s}\left(\overline{B_{\lambda}}\right)$,

$$
\left\|f(\lambda \cdot)\left|B_{p, q}^{s}\left(\mathbb{R}^{n}\right)\left\|\sim \lambda^{s-\frac{n}{p}}\right\| f\right| B_{p, q}^{s}\left(\mathbb{R}^{n}\right)\right\|
$$

where the equivalence constants are independent of $\lambda$ and $f$.

Proof. Let us fix $0<\lambda \leq 1$ and $f \in \widetilde{B}_{p, q}^{s}\left(\overline{B_{\lambda}}\right)$. We remark that $f, f(\lambda \cdot) \in \widetilde{B}_{p, q}^{s}\left(\overline{B_{1}}\right)$. By Proposition 5

(31) $\quad\left\|f(\lambda \cdot) \mid B_{p, q}^{s}\left(\mathbb{R}^{n}\right)\right\| \sim\left(\int_{\mathbb{R}^{n}}|h|^{-s q}\left\|\Delta_{h}^{M}(f(\lambda \cdot)) \mid L_{p}\left(\mathbb{R}^{n}\right)\right\|^{q} \frac{d h}{|h|^{n}}\right)^{1 / q}$

uniformly in $\lambda$. One inserts

$$
\Delta_{h}^{M}(f(\lambda \cdot))(x)=\left(\Delta_{\lambda h}^{M} f\right)(\lambda x)
$$

in $(31)$ and gets $(q<\infty)$

$$
\begin{aligned}
\left\|f(\lambda \cdot) \mid B_{p, q}^{s}\left(\mathbb{R}^{n}\right)\right\|^{q} & \sim \lambda^{-\frac{n q}{p}} \int_{\mathbb{R}^{n}}|h|^{-s q}\left\|\Delta_{\lambda h}^{M} f \mid L_{p}\left(\mathbb{R}^{n}\right)\right\|^{q} \frac{d h}{|h|^{n}} \\
& \sim \lambda^{q\left(s-\frac{n}{p}\right)} \int_{\mathbb{R}^{n}}|h|^{-s q}\left\|\Delta_{h}^{M} f \mid L_{p}\left(\mathbb{R}^{n}\right)\right\|^{q} \frac{d h}{|h|^{n}} \\
& \sim \lambda^{q\left(s-\frac{n}{p}\right)}\left\|f \mid B_{p, q}^{s}\left(\mathbb{R}^{n}\right)\right\|^{q}
\end{aligned}
$$

If $q=\infty$ one has to modify in the usual way. 


\section{Relations to Hardy inequalities}

Denoting the right-hand side of (10) by $\left\|f \mid b_{p, q}^{s}\left(\mathbb{R}^{n}\right)\right\|$ (which makes sense for all $0<p \leq \infty, 0<q \leq \infty, 0<s<M \in \mathbb{N}$ ) it follows from (6) (omitting $M$ on the left-hand side) for $\lambda>0$ that

$$
\left\|f(\lambda \cdot)\left|B_{p, q}^{s}\left(\mathbb{R}^{n}\right)\left\|=\lambda^{-\frac{n}{p}}\right\| f\right| L_{p}\left(\mathbb{R}^{n}\right)\right\|+\lambda^{s-\frac{n}{p}}\left\|f \mid b_{p, q}^{s}\left(\mathbb{R}^{n}\right)\right\| .
$$

Then the equivalence (30) can be reduced to the Hardy inequality

$$
\left\|f\left|L_{p}\left(\mathbb{R}^{n}\right)\left\|\leq c \lambda^{s}\right\| f\right| B_{p, q}^{s}\left(\mathbb{R}^{n}\right)\right\|, \quad f \in \widetilde{B}_{p, q}^{s}\left(\overline{B_{\lambda}}\right),
$$

where $c>0$ is independent of $f$ and of $0<\lambda<1$. Inequalities of this type attracted a lot of attention over the years. An early paper related to the above problems is [4]. More recent results (also for weighted counterparts) and references may be found in $[2,3]$.

Acknowledgements 1. The second named author is supported by Fundação para a Ciência e a Tecnologia (FCT) and European Social Fund in the scope of Community Support Framework III. This research was also partially supported by Unidade de Investigação Matemática e Aplicações of Universidade de Aveiro through Programa Operacional 'Ciência, Tecnologia, Inovação' (POCTI) of FCT, cofinanced by the European Community Fund (FEDER).

2. The authors wish to thank Prof. V. Burenkov for careful reading and for pointing out the relations to Hardy inequalities. We incorporated his valuable comments at the end of the paper.

\section{References}

[1] O.V. Besov, V.P. Il'in and S.M. Nikol'skij, Integral Representations of Functions and Embedding Theorems (Russian), Moskva, Nauka, 1975, sec. ed., 1996. (English translation: New York, Wiley, 1978/79).

[2] V.I. Burenkov, Sharp estimates for integrals over small intervals for functions possessing some smoothness, In : 'Progress in Analysis. Proc. 3rd Intern. ISAAC Congress', World Scientific, Vol. I (2003), 45-56.

[3] V.I. Burenkov, M.L. Goldman, Hardy inequalities for moduli of continuity, Trudy Mat. Inst. Steklov 227 (1999), 92-108. [Engl. transl.: Proc. Steklov Inst. Math. 227 (1999), 73-86.]

[4] B. Kuttner, Some theorems of fractional derivatives, Proc. London Math. Soc. VIII, 12 (1953), 480-497. 
[5] S.M. Nikol'skij, Approximation of Functions of Several Variables and Embedding Theorems (Russian), Sec. ed., Moskva, Nauka, 1977. (First ed., Moskva, Nauka, 1969; English translation, Berlin, Springer, 1975).

[6] J. Peetre, New Thoughts on Besov Spaces, Duke Univ. Math. Series. Durham, Univ., 1976.

[7] H. Triebel, Theory of Function Spaces, Birkhäuser, Basel, 1983.

[8] H. Triebel, Theory of Function Spaces II, Birkhäuser, Basel, 1992.

[9] H. Triebel, The Structure of Functions, Birkhäuser, Basel, 2001.

[10] H. Triebel, Theory of Function Spaces III, Birkhäuser, Basel, 2006.

António M. Caetano and Sofia Lopes

Departamento de Matemática

Universidade de Aveiro

3810-193 Aveiro

Portugal

(E-mail : acaetano@mat.ua.pt)

(E-mail : slopes@mat.ua.pt)

Hans Triebel

Mathematisches Institut, Fakultät für Mathematik und Informatik

Friedrich-Schiller-Universität Jena

D-07737 Jena

Germany

(E-mail : triebel@minet.uni-jena.de)

(Received : April 2005) 


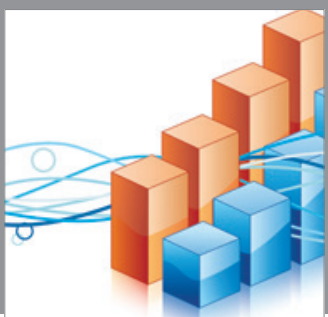

Advances in

Operations Research

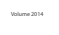

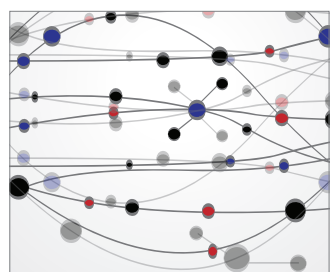

\section{The Scientific} World Journal
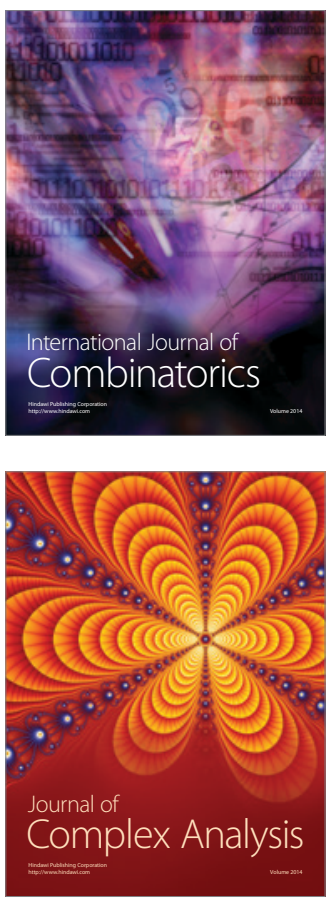

International Journal of

Mathematics and

Mathematical

Sciences
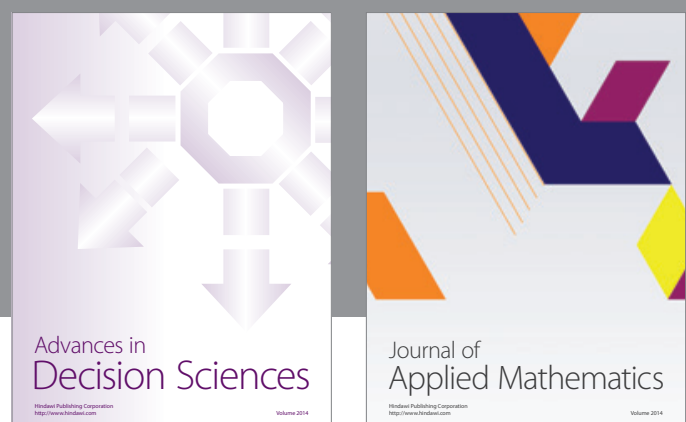

Journal of

Applied Mathematics
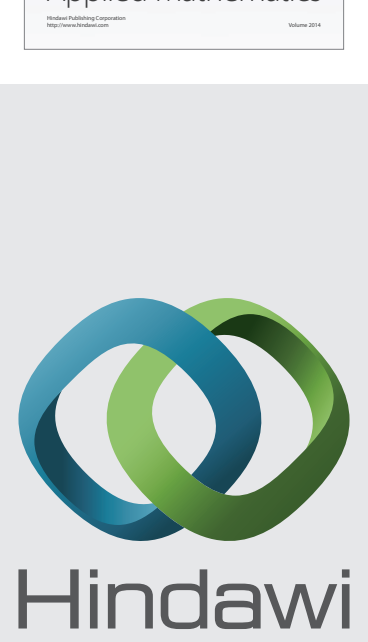

Submit your manuscripts at http://www.hindawi.com
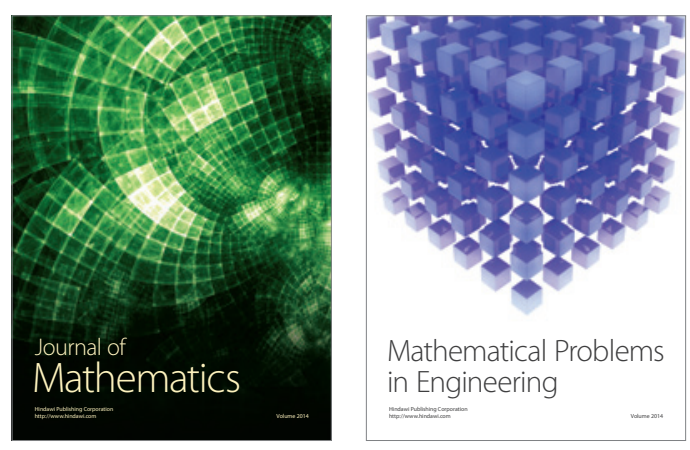

Mathematical Problems in Engineering
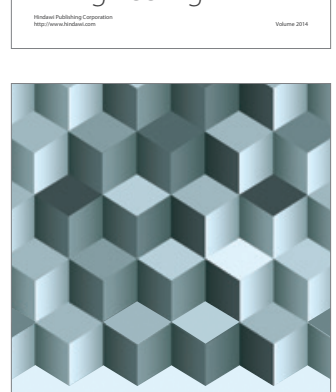

Journal of

Function Spaces
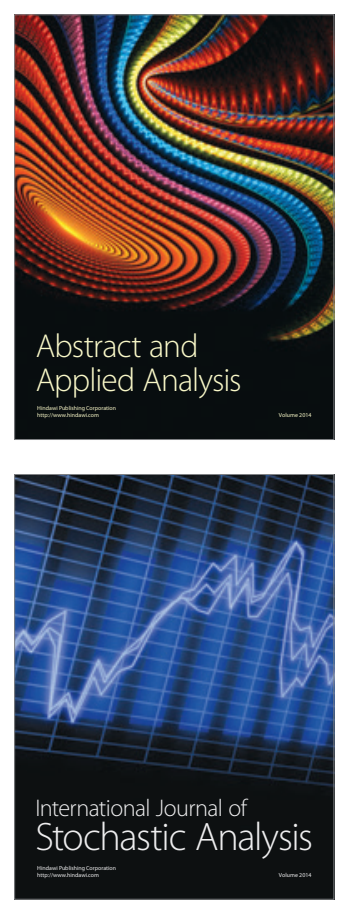

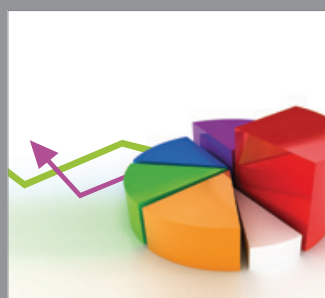

ournal of

Probability and Statistics

Promensencen
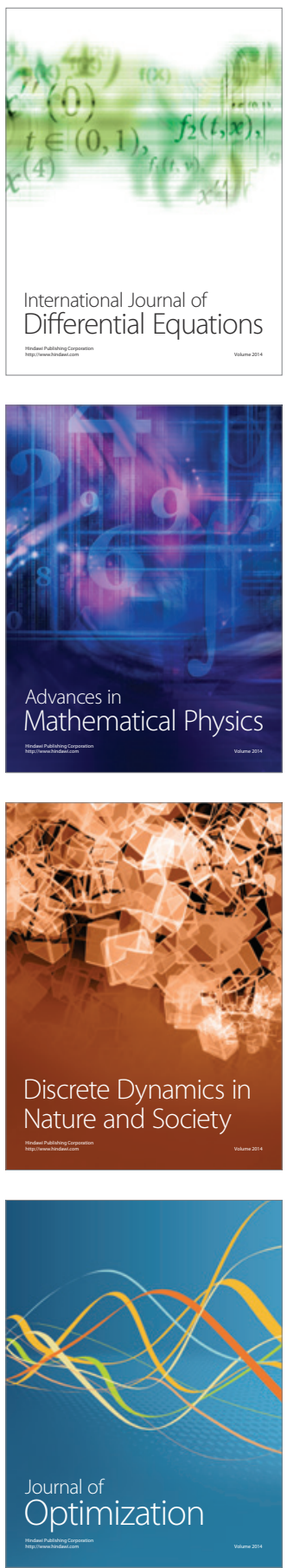